\title{
Thiol / disulfide balance and oxidative stress parameters in pediatric patients diagnosed with acute and chronic idiopathic thrombocytopenic purpura
}

\author{
Yeter Düzenli Kar ${ }^{1 \oplus}$, Zeynep Canan Özdemir ${ }^{2 \oplus}$, Özcan Bör ${ }^{2 \oplus}$ \\ ${ }^{1}$ Division of Pediatric Hematology, Department of Pediatrics, Afyonkarahisar Health Sciences University, Afyonkarahisar; ${ }^{2}$ Division of \\ Pediatric Hematology-Oncology, Department of Pediatrics, Eskişehir Osmangazi University Faculty of Medicine, Eskişehir, Turkey.
}

\begin{abstract}
Background. Changes in oxidative stress and thiol / disulfide balance are thought to play a role in the pathogenesis of idiopathic thrombocytopenic purpura (ITP). Our study investigates total oxidant level (TOS), total antioxidant level (TAS), oxidative stress index (OSI) levels and thiol / disulfide balance in pediatric patients with acute and chronic ITP.
\end{abstract}

Methods. Thirty four patients with acute ITP, eighteen patients with chronic ITP and thirty three healthy children (control) were included. TOS, TAS, OSI, thiol / disulfide balance were analyzed.

Results. In acute ITP, TAS levels were lower than chronic ITP and control, TOS and OSI levels were higher than control, and native thiol level was lower than chronic ITP ( $\mathrm{p}<0.05)$. In acute ITP; disulfide level, disulfide / native thiol and disulfide / total thiol ratios were higher than chronic ITP and control, and native thiol / total thiol ratio was lower than chronic ITP and control group $(p=0.038, p=0.018$, respectively). TOS and OSI levels of the chronic ITP were higher than the control group $(\mathrm{p}<0.05)$.

Conclusions. The results of this study have shown that oxidative stress increases in children with acute ITP and chronic ITP, that thiol / disulfide balance is disrupted in favor of disulfide in acute ITP, and that thiol / disulfide balance isn't disrupted in chronic ITP patients whose platelet count is close to normal and who don't require treatment.

Key words: acute, chronic, ITP, oxidative stress, TAS, TOS, thiol / disulfide balance.

Immune thrombocytopenia is an autoimmune disease characterized by isolated thrombocytopenia and defined as a platelet count below $100000 / \mathrm{mm}^{3}$ when the number of hemoglobin and white blood cells is normal. In approximately $80 \%$ of patients, the platelet count is below $30000 / \mathrm{mm}^{3}$. It commonly occurs between the ages of 2-5 years, and its annual incidence is reported to be 1.96.4 per 100,000 children. ${ }^{2}$ Etiopathogenesis is caused by a complex and multifactorial

Yeter Düzenli Kar

yeterduzenli@yahoo.com

Received 12th October 2020, revised 17th December 2020, 18th December 2020, 16th March 2021, 27th March 2021, accepted 31st March 2021. immune dysregulation targeting platelets and megakaryocytes. ${ }^{3,4}$ The best known mechanism is that immunoglobulin G (IgG) type autoantibody coated platelets formed against platelet surface antigens is phagocytized by the macrophages in the spleen via the $\mathrm{Fc}$ receptor, leading to shortened platelet life. In recent years, it has been shown that megakaryocyte inhibition develops in the bone marrow due to T-cell-mediated cytotoxicity resulting from immune dysregulation. ${ }^{5}$

In recent years, there are notable studies suggesting that oxidative stress is the mechanism that induces the disease. In idiopathic thrombocytopenic purpura (ITP) patients, it has been shown that gene expression pathways associated with oxidative stress are 
activated, and that higher level of oxidative stress and lower reduced glutathione / oxidized glutathione (GSH / GSSG) ratios are present compared to healthy controls. In addition, as a result of oxidative damage, it has been shown that patients with ITP develop lipid peroxidation and protein modification. ${ }^{6}$ Studies have reported that total oxidant level (TOS) and oxidative stress index (OSI) values are higher and total antioxidant level (TAS) is lower in both acute ITP and chronic ITP patients compared to healthy control groups. ${ }^{7,8}$

Thiols are organic compounds consisting of a sulfur atom and a hydrogen atom attached to a carbon atom and containing a sulfhydryl group $(-\mathrm{SH})$. While thiols in plasma are primarily composed of albumin and proteins, a few are composed of thiols with low molecular weight such as cysteine, cysteinylglycine, glutathione, homocysteine and $\gamma$-glutamyl cysteine. ${ }^{9}$ Oxidative products such as reactive oxygen species - which occur when oxidative stress increases - oxidize thiol groups to form disulfide bonds. As a result, dynamic thiol / disulfide homeostasis is achieved, and the organism is protected from the damage of reactive oxygen products. ${ }^{10}$

This study investigated TAS, TOS, OSI, total thiol and native thiol levels and the quantity of dynamic disulfide bonds in acute ITP, chronic ITP and control groups, and the relationship between these components was investigated.

\section{Material and Methods}

\section{Patient population}

Thirty-four children diagnosed with acute ITP (acute ITP group) and eighteen children diagnosed with chronic ITP (chronic ITP group) between May 2018 and August 2019 at the Pediatric Hematology and Oncology Clinic of Eskişehir Osmangazi University, as well as thirty-three healthy children (control group) were included in the study (date: 18.04.2018, no: $80558721 / 50)$.
The diagnosis of acute ITP was established based on clinical, laboratory and bone marrow findings and platelet count (platelet count $<100,000$ / $\mathrm{mm}^{3}$ ). Patients whose thrombocytopenia continued for three months from the time of diagnosis were classified as acute ITP, while those with thrombocytopenia continuing for 3-12 months after diagnosis were classified as persistent ITP. Chronic ITP patients consisted of patients with at least 12 months since the time of diagnosis and who had ongoing isolated thrombocytopenia. ${ }^{3}$ Approval was obtained from the ethics committee (date: 18.04.2018, no: $80558721 / 50$ ) and informed consent from legal guardians of the children were obtained.

\section{Exclusion criteria}

Patients with cytopenia, hepatomegaly, splenomegaly in other series in addition to thrombocytopenia; patients who had infection and vaccination one to four weeks before diagnosis; patients who had positive viral cereology results (hepatitis, EBV, CMV); and patients diagnosed with collagen tissue disease were excluded from the study. ITP patients using thrombopoietin mimetics were excluded from the study.

\section{Collection of blood samples}

Blood samples were collected from chronic ITP patients who did not receive any treatment (intravenous immunoglobulin, corticosteroid) in the past three months. Patients' age, gender, complete blood count parameters (hemoglobin, hemotocrit, red cell distribution width (RDW), platelet count) and C-reactive protein (CRP) values were recorded based on the information in patient files.

\section{Biochemical analysis}

Peripheral blood samples were transferred into biochemistry tubes. Serum was separated by centrifuging the blood samples at $2-8{ }^{\circ} \mathrm{C}$ for 10 minutes at $1500 \times$ g. Samples were stored at -80 ${ }^{\circ} \mathrm{C}$ until use. The levels of TOS, TAS, OSI, thiol / disulfide and [diannik] disulfide bonds were analyzed in the serum samples. 
TAS and TOS were measured spectrophotometrically using Rel Assay Diagnostics Kit (Rel Assay®, Diagnostics kits, Mega Tıp, Gaziantep, Turkey), at $660 \mathrm{~nm}$ for TAS and at $530 \mathrm{~nm}$ for TOS. OSI was calculated by proportioning the TOS values of the samples to the TAS values in terms of percentage. ${ }^{11-15}$ Reducible disulfide bonds were reduced to form free functional thiol groups. Formaldehyde was used to remove residual sodium borohydride and DTNB (5,5'-dithiobis- (2-nitrobenzoic acid)) products. Following this, both reduced and native thiol and natural thiol groups were determined. The quantity of dynamic disulfide bonds were calculated by dividing the difference between the total thiol and the native thiol groups by two. After calculating the quantity of native, total thiol and disulfide; the disulfide / total thiol percentage rates, the native thiol / total thiol percentage rates and the disulfide / native thiol percentage rates were also calculated. ${ }^{9}$

\section{Statistical analysis}

The data were statistically analyzed using the SPSS ver. 17.0 (SPSS Inc., Chicago, IL, USA) program package. All data were expressed as means \pm SD, medians (min-max) or numbers. Mann-Whitney $U$ test following Kruskall Wallis were used for comparison of the non parametric variables when appropriate. Oneway analysis of variance (ANOVA) was used to compare normally distributed variables. Post hoc Bonferroni and Tamhane's tests were used according to homogeneity of variances. The Chisquare was used to test the association between categorical outcome variables. Spearman rank correlation test was used for analysis of correlations among results. The $p$ value was set at 0.05 .

\section{Results}

Thirty-four children were included in the acute ITP group, 18 children in the chronic ITP group, and 33 children in the control group. There was no statistical difference in gender distribution between the three groups ( $p>0.05)$. Age of the acute ITP group was statistically younger than the chronic ITP group $(p=0.018)$. In acute ITP group, hemoglobin, hematocrit and platelet values were lower compared to the chronic ITP and control groups ( $p<0.05)$, and CRP values were higher $(p=0.048, p<0.001)$. Platelet values of the chronic ITP group were lower compared to the control group $(\mathrm{p}<0.001)$. The characteristic features and laboratory parameters of the patient and control groups are shown in Table I.

In the acute ITP group, TAS level was lower compared to the values of the chronic ITP and control groups $(\mathrm{p}=0.012, \mathrm{p}=0.001$, respectively), TOS and OSI levels were higher compared to the values of the control group ( $\mathrm{p}<0.001$, for both values), and native thiol level was lower compared to the values of the chronic ITP group $(p=0.025)$. In the acute ITP group, disulfide level was higher compared to the control group $(\mathrm{p}=0.007)$, while disulfide / native thiol and disulfide / total thiol ratios were higher compared to the values of the chronic ITP and control groups ( $p=0.038, p=0.018$, for both ratios), and native thiol / total thiol ratio was lower compared to that of the chronic ITP and control groups $(\mathrm{p}=0.038, \mathrm{p}=0.018)$.

TOS and OSI levels of the chronic ITP group were higher compared to the values of the control group $(\mathrm{p}<0.001, \mathrm{p}=0.005)$. TAS, native thiol, total thiol, disulfide levels, disulfide / native thiol, disulfide / total thiol and native thiol / total thiol ratio were similar to those of the control group ( $\mathrm{P}>0.05$ for all). There was no difference in total thiol level between the three groups. TOS, TAS, OSI level and thiol / disulfide balance parameters of the patient and control groups are shown in Table II.

There was a positive correlation between platelet count and TAS level in the patient group (acute and chronic ITP) $(\mathrm{r}=0.399, \mathrm{p}=0.014)$ and a negative correlation between platelet count and disulfide / native thiol, disulfide / total thiol ratio $(r=-0.912, p=0.001$, for both parameters).

While 27 of the patients diagnosed with acute ITP went into remission, seven of them became 
Table I. Demographic and laboratory data of the children with acute/chronic immune thrombocytopenia and of healthy controls.

\begin{tabular}{|c|c|c|c|c|}
\hline & Acute ITP & Chronic ITP & Healthy Controls & P Value \\
\hline \multirow{3}{*}{$\begin{array}{l}\text { Age (year) } \\
(\text { mean } \pm \text { SD) }\end{array}$} & $7.8 \pm 3.7$ & $10.9 \pm 3.7$ & $9.5 \pm 3.9$ & $0.018^{\alpha}$ \\
\hline & & & & $0.255^{\beta}$ \\
\hline & & & & $0.547^{\mathrm{T}}$ \\
\hline Sex (Female/male) (n) & $15 / 19$ & $11 / 7$ & $15 / 18$ & $0.466^{\alpha, \beta, \tau}$ \\
\hline \multirow{3}{*}{$\begin{array}{l}\operatorname{Hemoglobin}(\mathrm{g} / \mathrm{dl}) \\
(\text { mean } \pm \text { SD) }\end{array}$} & $12.6 \pm 0.9$ & $13.2 \pm 0.9$ & $12.9 \pm 0.95$ & $0.004^{\alpha}$ \\
\hline & & & & $0.020^{\beta}$ \\
\hline & & & & $0.996^{\mathrm{T}}$ \\
\hline \multirow{3}{*}{$\begin{array}{l}\text { Hematocrit }(\%) \\
(\text { mean } \pm \text { SD) }\end{array}$} & $37.4 \pm 2.53$ & $38.6 \pm 2.57$ & $38.7 \pm 3$ & $<0.001^{\alpha}$ \\
\hline & & & & $0.004^{\beta}$ \\
\hline & & & & $0.546^{\mathrm{T}}$ \\
\hline $\begin{array}{l}\text { RDW (\%) } \\
(\text { mean } \pm \text { SD) }\end{array}$ & $13.4 \pm 0.9$ & $13.2 \pm 0.9$ & $13.3 \pm 0.8$ & $0.247^{\alpha, \beta, \tau}$ \\
\hline $\begin{array}{l}\text { Platelet count }\left(/ \mathrm{mm}^{3}\right) \\
\text { Median (range) }\end{array}$ & $\begin{array}{c}9000 \\
(1000-21000)\end{array}$ & $\begin{array}{c}91000 \\
(34000-96000)\end{array}$ & $\begin{array}{c}345000 \\
(178000-490000)\end{array}$ & $<0.001^{\alpha, \beta, \mathrm{T}}$ \\
\hline CRP (mg/L) & 0.5 & 0.3 & 0.3 & $0.048^{\alpha}$ \\
\hline \multirow[t]{2}{*}{ Median (range) } & $(0.12-7)$ & $(0.2-1.5)$ & $(0.2-0.6)$ & $<0.001^{\beta}$ \\
\hline & & & & $0.124^{\mathrm{T}}$ \\
\hline
\end{tabular}

${ }^{\alpha}$ Acute ITP-Chronic ITP, ${ }^{\beta}$ Acute ITP - Healthy Controls, ${ }^{\mathrm{T}}$ Chronic ITP- Healthy Controls RDW; red cell distribution width, CRP; C-reaktive protein.

chronic. No statistically significant difference was found between the Thiol-disulfide balance and TAS, TOS, and OSI levels of patients who became chronic and in remission after one year of follow-up after the diagnosis of acute ITP in Table III.

\section{Discussion}

Although the pathogenesis of ITP has not yet been elucidated, increased platelet destruction and decreased platelet production due to loss of immune tolerance to platelet antigens are thought to play a role in the etiopathogenesis. In addition, the immune effects on both platelets and megakaryocytes due to the increase in T-cell-mediated cytotoxicity and the increase in $\mathrm{T}$ helper 17 proinflammatory cytokine are thought to play a role in the etiopathogenesis of ITP. ${ }^{16}$ A strong relationship between autoimmunity and increased oxidative stress has also been demonstrated in ITP. ${ }^{17}$ Free radicals, which normally occur as a product of physiological events in our body, are in a certain balance with antioxidants. 6 There are studies suggesting that oxidative stress triggers autoimmunity in both acute and chronic ITP, leading to impairment in platelet structure and platelet dysfunction. ${ }^{4,7,8,16,18,19}$ It has also been reported that oxidative stress plays a role in acute ITP becoming chronic. ${ }^{19}$

The results of our study show that TOS and OSI levels are higher in acute and chronic ITP patients compared to the control group, and that TAS level in acute ITP patients is lower compared to the chronic ITP and control groups. Cura et al. ${ }^{7}$ found that TAS level was lower and TOS and OSI levels were significantly higher in acute ITP patients compared to the control group. Akbayram et al. ${ }^{8}$ reported that they could not detect a significant difference in TAS levels between acute and chronic ITP patients, and that TAS level decreased in both acute ITP and chronic ITP groups compared to healthy controls. In the same study, they found that in cases where oxidative stress and TOS and OSI 
Table II. Thiol-disulfide balance and TAS, TOS, OSI levels of children diagnosed with acute and chronic idiopathic thrombocytopenic purpura and healthy control group.

\begin{tabular}{|c|c|c|c|c|}
\hline & Acute ITP & Chronic ITP & Healthy control & $\mathrm{P}$ value \\
\hline TAS ( $\mu$ mol Trolox Eqv./L) & 1.23 & 1.34 & 1,38 & $0.012^{\alpha}$ \\
\hline \multirow[t]{2}{*}{ Median (Lower-upper limit) } & $(0.21-2)$ & $(0.95-2.06)$ & $(1.09-2.020)$ & $0.001^{\beta}$ \\
\hline & & & & $0.805^{\mathrm{T}}$ \\
\hline TOS ( $\mu$ mol H2O2 Eqv./L) & 30 & 32,6 & 12,9 & $0.489^{\alpha}$ \\
\hline Median (Lower-upper limit) & $(9.55-77.8)$ & $(11.1-70.6)$ & $(1.1-58.8)$ & $<0.001^{\beta, \tau}$ \\
\hline OSI (U/L) & $1.86(0.57-5.93)$ & $1.51(0.78-4.22)$ & $1.06(0.001-5.07)$ & $0.248^{\alpha}$ \\
\hline \multirow[t]{2}{*}{ Median (Lower-upper limit) } & & & & $<0.001^{\beta}$ \\
\hline & & & & $0.005^{\mathrm{T}}$ \\
\hline Native thiol $(\mu \mathrm{mol} / \mathrm{L})$ & 398(114-635) & $444(299-600)$ & $428(311-591)$ & $0.025^{\alpha}$ \\
\hline \multirow[t]{2}{*}{ Median (Lower-upper limit) } & & & & $0.079^{\beta}$ \\
\hline & & & & $0.344^{\mathrm{T}}$ \\
\hline Total thiol $(\mu \mathrm{mol} / \mathrm{L})$ & $566(440-792)$ & $565(473-678)$ & $533(480-659)$ & $0.254^{\alpha, \beta, \tau}$ \\
\hline \multicolumn{5}{|l|}{ Median (Lower-upper limit) } \\
\hline Disulfide $(\mu \mathrm{mol} / \mathrm{L})$ & $79.7(10-237)$ & $59(6-113)$ & $58.5(3-138.5)$ & $0.074^{\alpha}$ \\
\hline \multirow[t]{2}{*}{ Median (Lower-upper limit) } & & & & $0.007^{\beta}$ \\
\hline & & & & $0.730^{\mathrm{T}}$ \\
\hline Disulfide/ nativ thiol (\%) & $20.9(1.91-206.5)$ & $13.4(1-34)$ & $12,9(0.6-43.4)$ & $0.038^{\alpha}$ \\
\hline \multirow[t]{2}{*}{ Median (Lower-upper limit) } & & & & $0.018^{\beta}$ \\
\hline & & & & $0.953^{\mathrm{T}}$ \\
\hline Disulfide/ total thiol (\%) & $14.7(1.8-40.2)$ & $10,5(0,9-20,4)$ & $10.3(0.6-23.2)$ & $0.038^{\alpha}$ \\
\hline \multirow[t]{2}{*}{ Median (Lower-upper limit) } & & & & $0.018^{\beta}$ \\
\hline & & & & $0.953^{\mathrm{T}}$ \\
\hline Nativ thiol/ total thiol (\%) & 70.4(19.4-96.3) & 78.8(59-98) & 79.4(53.5-98.8) & $0.038^{\alpha}$ \\
\hline \multirow[t]{2}{*}{ Median (Lower-upper limit) } & & & & $0.018^{\beta}$ \\
\hline & & & & $0.953^{\mathrm{T}}$ \\
\hline
\end{tabular}

$\alpha$ Acute ITP-Chronic ITP, ${ }^{\beta}$ Acute ITP - Healthy Controls, ${ }^{T}$ Chronic ITP- Healthy Controls

TOS: total oxidant level, TAS: total antioxidant level, OSI: oxidative stress index

values increased, the level of Malondialdehyde - which is a good indicator of lipid peroxidation - increased in both acute ITP and chronic ITP patients compared to the control group. Elalfy et al. ${ }^{4}$ showed that the reduced glutathione, TAS and catalase (known to be antioxidant systems within the organism) decreased in the acute ITP and chronic ITP groups compared to healthy controls, while the level of malondialdehyde, a lipid peroxidation product, increased. The reason for the difference in TAS level between our chronic ITP group and the control group may be that the number of patients in the chronic ITP group was low and that these patients are composed of patients with close to normal platelet counts who did not require treatment.

Thiols are molecules containing sulfhydryl group $(-\mathrm{SH})$ that can be converted into reversible disulfide bond structures through oxidation by oxidant molecules such as free radicals or reactive oxygen species. This conversion is the earliest indicator of protein oxidation in case of oxidative stress. Disulfide bond structures formed can be reduced to thiol groups again, thus ensuring the thioldisulfide balance. ${ }^{9}$ Disulfide formation can be used as a reliable indicator of oxidative stress, since it reflects the antioxidant status, prooxidant reactions and protein-thiol redox 
Table III. Thiol-disulfide balance and TAS, TOS, OSI levels of children diagnosed with acute ITP in remission and acute ITP that becomes chronic group.

\begin{tabular}{|c|c|c|c|}
\hline & In remission acute ITP & Acute ITP that becomes chronic group & $\mathrm{p}$ value \\
\hline Age (year) & $7.04 \pm 3.5$ & $11.07 \pm 2.1$ & 0.008 \\
\hline \multicolumn{4}{|l|}{$($ mean \pm SD) } \\
\hline Sex (Female/male) (n) & $10 / 17$ & $5 / 2$ & 0.199 \\
\hline TAS ( $\mu$ mol Trolox Eqv./L) & $1.22(0.21-1.48)$ & $1.27(1.13-2)$ & 0.259 \\
\hline \multicolumn{4}{|l|}{ Median (Lower-upper limit) } \\
\hline TOS ( $\mu$ mol H2O2 Eqv./L) & 29.71(6.44-77.8) & $35.22(19.04-41.04)$ & 0.594 \\
\hline \multicolumn{4}{|l|}{ Median (Lower-upper limit) } \\
\hline OSI (U/L) & $1.87(0.57-5.93)$ & $1.68(1.25-3.46)$ & 0.686 \\
\hline \multicolumn{4}{|l|}{ Median (Lower-upper limit) } \\
\hline \multicolumn{3}{|l|}{$(\mathrm{mean} \pm \mathrm{SD})$} & 0.626 \\
\hline \multicolumn{3}{|l|}{$($ mean $\pm \mathrm{SD})$} & 0,418 \\
\hline Disulfide $(\mu \mathrm{mol} / \mathrm{L})$ & 78.5(10-237) & $86(46-173)$ & 0.624 \\
\hline \multicolumn{4}{|l|}{ Median (range) } \\
\hline Disulfide/ nativ thiol (\%) & $0.22(0.02-2.07)$ & $0.16(0.1-0.64)$ & 0.983 \\
\hline \multicolumn{4}{|l|}{ Median (range) } \\
\hline $\begin{array}{l}\text { Disulfide/ total thiol (\%) } \\
(\text { mean } \pm \text { SD) }\end{array}$ & $0.15 \pm 0.09$ & $0.15 \pm 0.06$ & 0.948 \\
\hline $\begin{array}{l}\text { Nativ thiol/ total thiol }(\%) \\
(\text { mean } \pm \text { SD) }\end{array}$ & $0.68 \pm 0.19$ & $0.68 \pm 0.12$ & 0.948 \\
\hline
\end{tabular}

TAS: total antioxidant level, TOS: total oxidant level, OSI: oxidative stress index

status in the organism. ${ }^{20}$ It has been shown that plasma disulfide level is high in smokers and in degenerative diseases such as obesity, diabetes and pneumonia, and low in proliferative diseases such as multiple myeloma, colon and renal cancer, and aggressive growing tumors. ${ }^{9}$ In our study, there was no difference in total thiol between the three groups, while plasma native thiol levels were significantly lower in acute ITP patients compared to chronic ITP patients. Serum disulfide levels of acute ITP patients were statistically higher compared to the control group and chronic ITP group. Disulfide / native thiol (\%), disulfide / total thiol $(\%)$ and native thiol / total thiol (\%) ratios were statistically significantly higher in the acute ITP group compared to both the chronic ITP and the control groups. This indicates that during oxidative stress in the acute ITP group, thiols tend to be reduced and increase the formation of disulfide bonds, and the thiol-disulfide balance shifts towards disulfide direction. In our study, there was no statistically significant difference in disulfide / native thiol, disulfide / total thiol and native thiol / total thiol ratios between the chronic ITP group and the control group. In a recent study by Beyazit et al. ${ }^{18}$, in which they investigated the plasma thiol-disulfide balance before and after treatment in patients with acute ITP, they found that the pre-treatment plasma native and total thiol levels were lower in patients with acute ITP compared with the post-treatment values and the levels of the control group. The same study showed that the pre-treatment disulfide level was similar to the control group, while post-treatment disulfide level increased significantly compared to the pre-treatment level. 
In our study, there was no significant difference in native thiol and total thiol levels between the acute ITP and the control group. However, it was observed that thiol-disulfide balance shifted towards disulfide increase in acute ITP patients compared to the control group. There was a positive correlation between platelet counts and TAS, and a negative correlation between thiol / disulfide balance in the patient group.

Hemoglobin and hemotocrit values in the acute ITP group were lower compared to both the chronic ITP group and the control group. This result can be explained by the common prevalence of skin and mucosa bleeding such as petechiae, ecchymosis, epistaxis in acute ITP patients at diagnosis.

In our study, in accordance with the literature, the ages of the patients who were diagnosed with acute ITP and became chronic after a oneyear follow-up were found to be statistically greater than the patients in remission., However, there was no statistically significant difference in thiol-disulfide balance and oxidative stress parameters between patients who became chronic after the diagnosis of acute ITP and those who were in remission. We think that the reason for this is the low number of patients. There is a need for comprehensive studies on this subject.

The limitations of our study are that the number of patients was low and that the platelet counts of chronic ITP patients were close to normal.

In conclusion, the results of this study have shown that oxidative stress increases in children with both acute ITP and chronic ITP; that TAS level decreases significantly in acute ITP; that thiol / disulfide balance is disrupted in favor of disulfide; and that, compared to controls, the thiol / disulfide balance is not disrupted in chronic ITP patients with close to normal platelet counts who do not require treatment. It has been shown that the conversion of native thiol groups to reversible disulfide bonds is increased due to increased oxidative stress in the organism in order to maintain oxidative balance. We believe that showing the increased disulfide / thiol ratios in patients with acute ITP will contribute to elucidation of ITP etiopathogenesis and development of new treatment methods targeting the disulfide / thiol balance in addition to the currently used treatment methods.

\section{Acknowledgment}

This study was supported by the Eskişehir Osmangazi University Faculty of scientific research projects commission (project no: 2018/2154).

\section{Author contribution}

We require that all authors take responsibility for the content of the work submitted. An author contribution section should be given. The contributions of all authors must be described in the following manner: The authors confirm contribution to the paper as follows: study conception and design: YDK, ZCÖ, ÖB; data collection: YDK, ZCÖ; analysis and interpretation of results: YDK, ZCÖ; draft manuscript preparation: YDK. All authors reviewed the results and approved the final version of the manuscript.

\section{Ethical approval}

Approval was obtained from Eskişehir Osmangazi University Clinical Researches Ethics Committee (date: 18.04.2018, no: 80558721/50).

\section{Source of funding}

None.

\section{Conflict of interest}

No conflict of interest. 


\section{REFERENCES}

1. Petrovic D, Benzon B, Batinic M, Culic S, Roganovic J, Markic J. Hypovitaminosis D influences the clinical presentation of immune thrombocytopenia in children with newly diagnosed disease. J Clin Med 2019; 8: 1861. https://doi.org/10.3390/jcm8111861

2. Lee AC. Isolated thrombocytopenia in childhood: what if it is not immune thrombocytopenia? Singapore Med J 2018; 59: 390-393. https://doi. org/10.11622/smedj.2018089

3. Singh G, Bansal D, Wright NAM. Immune thrombocytopenia in children: consensus and controversies. Indian J Pediatr 2020; 87: 150-157. https://doi.org/10.1007/s12098-019-03155-4

4. Elalfy MS, Elhenawy YI, Deifalla S, Hegazy M, Sabra A, Abdelaziz Y. Oxidant/antioxidant status in children and adolescents with immune thrombocytopenia (ITP) and the role of an adjuvant antioxidant therapy. Pediatr Blood Cancer 2015; 62: 830-837. https://doi.org/10.1002/pbc.25434

5. Semple JW, Provean D. The immunopathogenesis of immune thrombocytopenia: T cells still take centerstage. Curr Opin Hematol 2012; 19: 357-362. https:// doi.org/10.1097/MOH.0b013e3283567541

6. Zhang B, Zehnder JL. Oxidative stress and immune thrombocytopenia. Semin Hematol 2013; 50: e1-e4. https://doi.org/10.1053/j.seminhematol.2013.06.011

7. Cura M, Koç A, Aksoy N, Özdemir ZC. Effect of short-term, high-dose methylprednisolone on oxidative stress in children with acute immune thrombocytopenia. Blood Res 2016; 51: 261-267. https://doi.org/10.5045/br.2016.51.4.261

8. Akbayram S, Doğan $M$, Akgün $C$, et al. The association of oxidant status and antioxidant capacity in children with acute and chronic ITP. J Pediatr Hematol Oncol 2010; 32: 277-281. https://doi. org/10.1097/MPH.0b013e3181d6421f

9. Erel O, Neselioglu S. A novel and automated assay for thiol/disulphide homeostasis. Clin Biochem 2014; 47: 326-332. https://doi.org/10.1016/j. clinbiochem.2014.09.026

10. Yıkılmaz AŞ, Bakanay ŞM, Akinci S, Alisik M, Erel Ö, Dilek İ. Thiol-disulphide homeostasis in essential thrombocythemia patients. J Med Biochem 2019; 38: 475-480. https://doi.org/10.2478/jomb-2018-0045
11. Erel O. A novel automated direct measurement method for total antioxidant capacity using a new generation, more stable ABTS radical cation. Clin Biochem 2004; 37: 277-285. https://doi.org/10.1016/j. clinbiochem.2003.11.015

12. Erel O. A new automated colorimetric method for measuring total oxidant status. Clin Biochem 2005; 38: 1103-1111. https://doi.org/10.1016/j. clinbiochem.2005.08.008

13. Yumru M, Savas HA, Kalenderoglu A, Bulut M, Celik $\mathrm{H}$, Erel O. Oxidative imbalance in bipolar disorder subtypes: a comparative study. Prog Neuropsychopharmacol Biol Psychiatry 2009; 33: 1070-1074. https://doi.org/10.1016/j. pnpbp.2009.06.005

14. Kosecik M, Erel O, Sevinc E, Selek S. Increased oxidative stress in children exposed to passive smoking. Int J Cardiol 2005; 100: 61-64. https://doi. org/10.1016/j.ijcard.2004.05.069

15. Harma M, Harma M, Erel O. Increased oxidative stress in patients with hydatidiform mole. Swiss Med Wkly 2003; 133: 563-566.

16. Jin CQ, Dong HX, Cheng PP, Zhou JW, Zheng BY, Liu F. Antioxidant status and oxidative stress in patients with chronic ITP. Scand J Immunol 2013; 77: 482-487. https://doi.org/10.1111/sji.12048

17. Kühne T. Diagnosis and management of immmune thrombocytopenia in childhood. Hamostaseologie 2017; 37: 36-44. https://doi.org/10.5482/HAMO-1606-0017

18. Beyazit H, Demiryürek AT, Temel MT, Pekpak E, Demiryürek S, Akbayram S. Investigation of dynamic thiol/disulfide homeostasis in children with acute immune thrombocytopenia. J Pediatr Hematol Oncol 2019; 41: 463-467. https://doi.org/10.1097/ MPH.0000000000001494

19. Elsalakawy WA, Ali MA, Hegazy MG, Farweez BA Value of vanin-1 assessment in adult patients with primary immune thrombocytopenia. Platelets 2014; 25: 86-92. https://doi.org/10.3109/09537104.2013.7824 84

20. Gul F, Muderris T, Yalciner G, et al. A novel method for evaluation of oxidative stress in children with OSA. Int J Pediatr Otorhinolaryngol 2016; 89: 76-80. https://doi.org/10.1016/j.ijporl.2016.07.035 